\title{
The Role of Vascular Injury and Congestion in the Pathogenesis of Cirrhosis: the Congestive Escalator and the Parenchymal Extinction Sequence
}

\author{
Ian R. Wanless ${ }^{1}$ \\ Published online: 28 January 2020 \\ (C) The Author(s) 2020
}

\begin{abstract}
Purpose of Review Current research into the pathogenesis of cirrhosis is largely dominated by investigations of hepatocellular injury and fibrogenesis, mostly in short-term experimental models. Cirrhosis in the human evolves for decades with histologic features that are very different from the models studied, dominated by hepatic vein obstruction and congestion. This is a clue that the mechanisms operating in the human are different from those in most animal models.

Recent Findings This paper presents an updated "vascular hypothesis" with previously unpublished observations that provide a more complete understanding of the pathogenesis of chronic liver disease in the human: (1) a definition of parenchymal extinction emphasizing the importance of sinusoidal destruction, (2) analysis of the temporal evolution of parenchymal extinction lesions, (3) new data to quantify hepatic vein obstruction, (4) a "congestive escalator" hypothesis to explain how vascular obstruction occurs, beginning with sinusoidal endothelial cell injury, fluid translocation, and vascular compression by mechanics known as "compartment syndrome," (5) a "nested cone model" of hepatic vein anatomy that predisposes to compartment syndrome in the human, and (6) a proposal for the mechanism of collagen formation in response to congestion ("congestive fibrosis").

Summary The guiding principle in this model is that flow has to be vented to keep pressure gradients within the physiological range. Vascular obstruction causes tissue congestion which induces further vascular obstruction that drives a congestive escalator leading to progressive parenchymal extinction. This model may be applicable to all types of cirrhosis found in the human.
\end{abstract}

Keywords Sinusoids $\cdot$ Sinusoidal endothelial cells $\cdot$ Parenchymal extinction $\cdot$ PELs $\cdot$ Congestive vasculopathy $\cdot$ Congestive escalator $\cdot$ Compartment syndrome $\cdot$ Buds $\cdot$ Regeneration $\cdot$ Regression $\cdot$ Non-cirrhotic portal hypertension

\section{Introduction}

Cirrhosis is defined anatomically by the presence throughout the liver of hepatocellular nodules that are separated by septa. The principle clinical manifestations are related to portal hypertension. It is widely accepted that cirrhosis is caused by a

This article is part of the Topical Collection on Portal Hypertension

Electronic supplementary material The online version of this article (https://doi.org/10.1007/s11901-020-00508-y) contains supplementary material, which is available to authorized users.

Ian R. Wanless

iwanless@eastlink.ca

1 Department of Pathology, Queen Elizabeth II Health Sciences Centre and Dalhousie University, Halifax, NS B3H 1V8, Canada primary injury that results in loss of hepatocytes followed by synthesis of collagen that accumulates in the septa. Portal hypertension is thought to result from vascular compromise in this distorted architecture [1]. Discussions of pathogenesis have usually concentrated on the mechanisms of liver cell injury and fibrogenesis [1-6]. The significance of vascular injury in the progression of liver disease is not widely appreciated, although there is new interest with evidence that anticoagulants, simvastatin, and VEGF modulation have protective effects on the liver $[7,8 \bullet \bullet, 9,10 \bullet, 11,12]$.

This paper presents an updated vascular hypothesis for the pathogenesis of cirrhosis. The original, proposed in 1994, suggested that regions of tissue loss and fibrosis (called parenchymal extinction) were secondary to vascular obstruction $[13,14 \bullet, 15]$. New observations were incorporated sequentially in several textbooks and elsewhere [16, 17, 18•]. The current model includes previously unpublished observations 


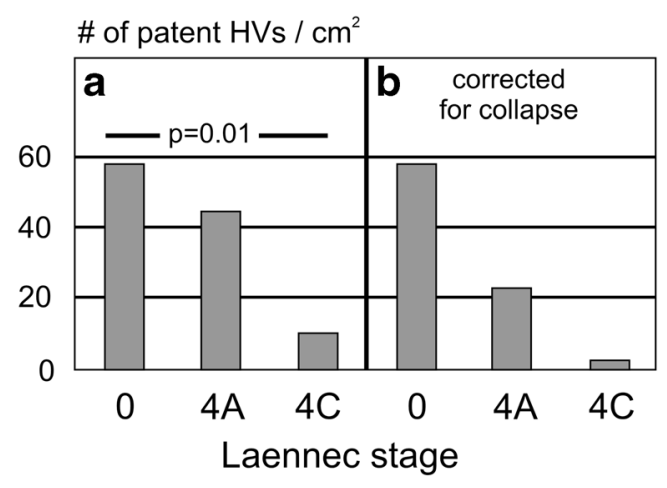

\# of HVs in septa or parenchyma $/ \mathrm{cm}^{2}$

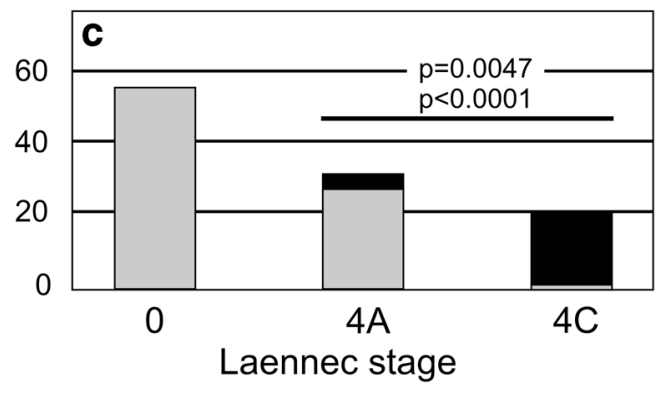

septa $\square$ parenchyma $\square$ all

Fig. 1 Morphometric analysis of hepatic veins versus stage. Hepatic veins were counted in large sections (area of tissue measured $\geq 1 \mathrm{~cm}^{2}$ ) from 8 cirrhotic explants (stage 4A (alcohol 2, HCV 3) and stage 4C (alcohol 1, HCV 2)) and 4 normal autopsy controls. All veins $\geq 20 \mu$ diameter were counted and were considered obstructed if the luminal area was $<50 \%$ of the vein profile. All portal tracts with a duct $\geq$ $50 \mu \mathrm{m}$ diameter were counted, as a measure of tissue collapse. Statistics were ANOVA test using Analyse-it 5.10.7. Significance with $p<0.05$ was confirmed using the Kruskal-Wallis test. a The density of patent hepatic veins declined from $57.9 / \mathrm{cm}^{2}$ in normal livers to $11.0 / \mathrm{cm}^{2}$ in stage 4C cirrhosis. b Because cirrhotic livers are collapsed and contain more portal tracts and hepatic veins than normal, the data was corrected for collapse by dividing the $\mathrm{HV}$ data by the fold increase in portal tracts compared to controls ( $1.9 x$ for $4 \mathrm{~A}, 3.4 x$ for $4 \mathrm{C}$ ). The corrected data gives an estimate of the percent loss of patent HVs of 59\% in 4A livers and $94 \%$ in $4 \mathrm{C}$ livers. c Hepatic veins were recorded as being located in parenchyma (nodules) or septa. In mild cirrhosis (4A), most visible HVs were in the nodules. However, in severe cirrhosis (4C), almost all visible HVs were in septa. This result indicates that, as cirrhosis progresses, tissue is destroyed and remaining structures, including portal tracts and $\mathrm{HVs}$, collapse into septa. In $4 \mathrm{~A}$ cirrhosis, where most parenchyma is in macro-nodules, the number of HVs per $\mathrm{cm}^{2}$ of parenchymal area is reduced a modest amount (28\%) compared to controls (not shown in graph). This indicates that macro-nodules have relatively normal $\mathrm{HV}$ drainage, especially noticeable in comparison with micro-nodules of $4 \mathrm{C}$. This finding can be explained by two origins of cirrhosis 4A. Some cases are mild because PELs (i.e., septum formation) was limited so there are regions whose architecture was never destroyed. The second type is moderate cirrhosis (likely 4B) that has regressed to 4A by repopulation of the septa with hepatocyte buds that did not have difficulty finding a patent $\mathrm{HV}$ for drainage. In contrast, the micronodules of $4 \mathrm{C}$ cirrhosis seldom contain $\mathrm{HVs}$ but are drained retrogradely into patent portal veins located in septa. This correlates with the high prevalence of retrograde PV flow in patients with severe cirrhosis
Fig. 2 Congestive injury in cirrhotic livers. a, b Gross appearance of cut surfaces of 2 cirrhotic livers (etiology: $\mathrm{HCV}$ in $\mathbf{a}$, alcohol in $\mathbf{b}$ ). a There is regional variation in nodule size and congestion. On the right, beneath the capsule, nodules are small, congested, and separated by broad septa. On the left, the nodules are larger, less congested, and surrounded by thinner septa. $\mathbf{b}$ In the second liver, at higher magnification, there is prominent atrophy and hemorrhage. Bar $=2 \mathrm{~mm}$. c-e Cirrhosis showing prominent "stress zones". c, $\mathbf{d}$ The tissue is variegated in color with pale parenchyma admixed with stress zones that are atrophic and darker in color. The darker color is largely due to cytoplasm that is depleted of glycogen and fat. The region in the box (enlarged in $\mathbf{d}$ ) shows that necrosis and hemorrhage are found only in stress zones. e Under higher magnification, a stress zone shows hepatocellular atrophy, sinusoidal dilatation, and two hepatic veins with lumina obstructed by collagen fibers admixed with red cells. The lower vein is in the process of recanalizing. $\mathbf{f}-\mathbf{j}$ In this liver, there is marked regional variation in severity (Laennec 4B in $\mathbf{f}$ and $\mathbf{g}$, Laennec $4 \mathrm{C}$ in $\mathbf{h}-\mathbf{j})$. f, $\mathbf{g}$ In the milder region, congestive changes are mild, with stress zones showing focal sinusoidal dilatation and hepatocellular atrophy. At higher magnification in $\mathbf{g}$, boxes identify certain features. Tissue in the left box shows sinusoidal dilatation and congestion, hepatocellular atrophy, endothelial cell lifting and loss. The venules and artery have been dissected from the hepatocellular plates by red blood cells. The right box shows a venule with endothelial lifting and luminal obstruction. $\mathbf{h}-\mathbf{j}$ In the severe region, the regenerative nodules are very small and separated by broad septa (tissue in boxes are enlarged in $\mathbf{i}$ and $\mathbf{j}$ ). The nodules are severely congested. All hepatic veins visible at this magnification are partially obstructed (arrows, enlarged in $\mathbf{j}$ ). $\mathbf{i}$ This nodule is partially necrotic with early collapse to form several smaller nodules. j A large HV has marked luminal compromise by intimal edema and fibrosis. $\mathbf{k}$ Large hepatic vein with focal intimal fibrosis (arrow). The pathogenesis of intimal fibrosis of large hepatic veins can be understood from this photograph. These veins are physically remote from the primary disease, but in severe cirrhosis the adventitia is surrounded by a large region of parenchymal extinction containing many arteries, dilated veins, and lymphatics, some of which have herniated into the media and intima. At these points, a high transmural pressure gradient favors transudation of fluid that organizes into intimal fibrosis. I Severe cirrhosis (Laennec 4C) with marked fibrous thickening of the hepatic capsule. The patient required frequent paracentesis for ascites prior to transplantation. The mechanism of capsular fibrosis is likely the same as described in $\mathbf{k}$. (Etiology: $\mathrm{HCV}$ in a, $\mathrm{HCV}$ plus alcohol in $\mathbf{e}$, alcohol in all others. Patients with oxaliplatin exposure were excluded. All sections were stained with elastic trichrome.)

that provide a more complete and detailed understanding of the pathogenesis of chronic liver disease and progression to cirrhosis. The definition of parenchymal extinction [14••] is modified to recognize the importance of sinusoidal destruction, including loss of sinusoidal endothelial cells (SEC). The temporal evolution of histologic features is incorporated into a "parenchymal extinction sequence". New data to quantify hepatic vein (HV) obstruction is provided. The "congestive escalator" hypothesis $[16,19]$ is further elaborated to explain how vascular obstruction begins with onset of SEC injury, fluid translocation, and vascular compression by mechanics well-known in other organs as "compartment syndrome." A "nested cone model" of hepatic vein anatomy is described that facilitates the development of a compartment syndrome effect. A mechanism of collagen formation in response to congestion ("congestive fibrosis") is proposed. Arterialization (capillarization) is also discussed. 

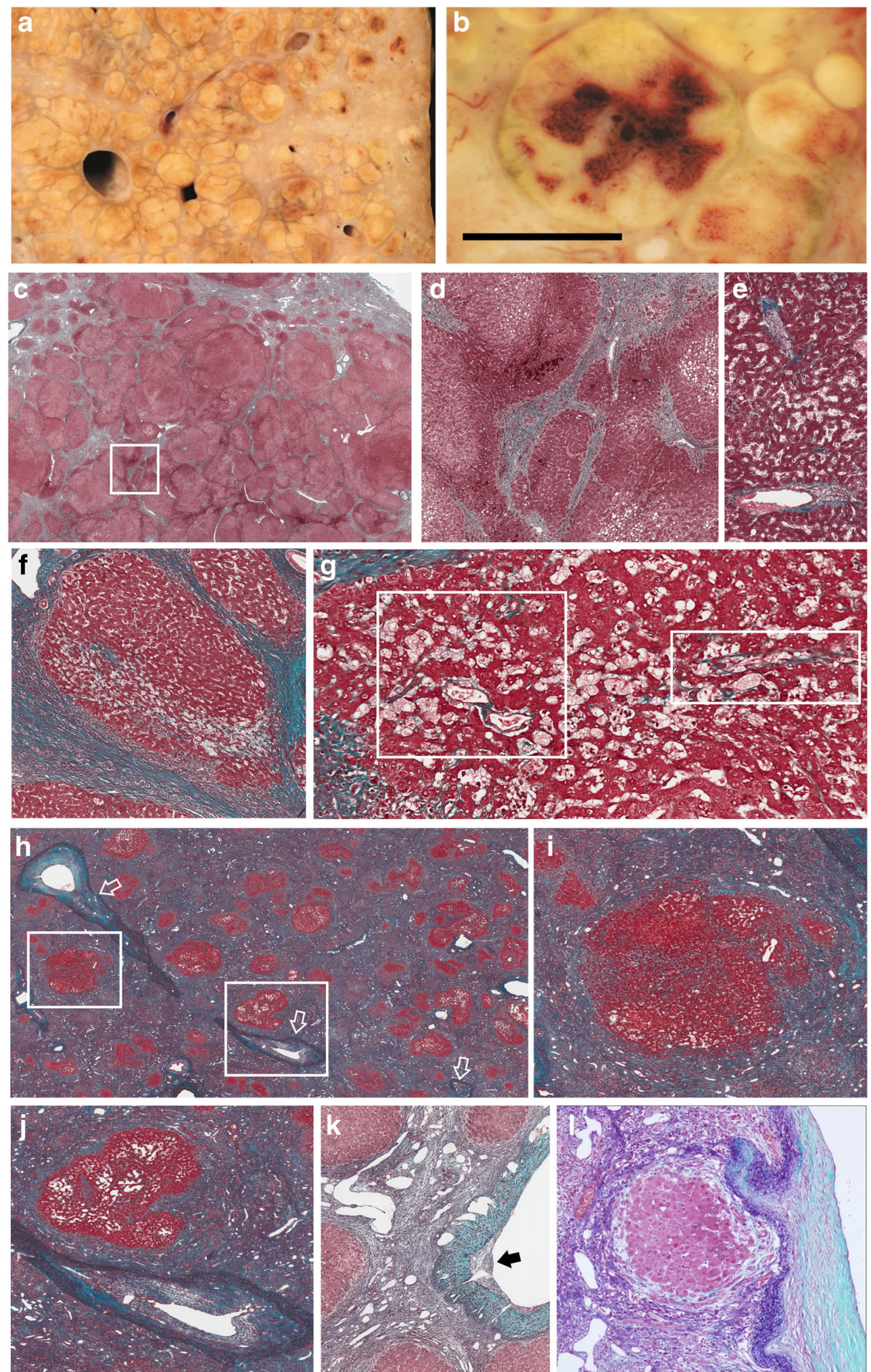

The Congestive Escalator The pathogenesis of chronic liver disease can be summarized with the concept of a "congestive escalator," whereby vascular injury causes vascular obstruction which causes more vascular injury which causes more vascular obstruction, in a positive feedback loop. This mechanism operates because vascular obstruction increases tissue pressure gradients which drive transudation of fluid into vessel walls and interstitial tissues to 
cause vascular obstruction. Vascular obstruction causes hepatocellular ischemia followed by parenchymal extinction lesions (PELs) with collapse of the architecture. Collapse and regeneration explain the tissue distortion found in cirrhosis. An important feature of the congestive escalator is that vascular injury progresses to cause obstructing lesions in ever larger branches of the hepatic and portal veins so that PELs become larger and eventually merge into confluent septa.

Although vascular injury is the central event in this paradigm, parenchymal extinction is more easily quantified in tissue sections. Therefore, the process of parenchymal extinction has been selected as the best focal point for analysis of the pathogenesis of cirrhosis and its regression.

Definition of Parenchymal Extinction Parenchymal extinction lesion was originally defined as a region with loss of contiguous hepatocytes [14••]. While loss of hepatocytes is the most obvious feature, loss of the local microvasculature is probably more significant for the progression of disease. A revised definition is "a region with focal loss of contiguous hepatocytes and adjacent microvascular structures." An important feature of PELs is that local vascular impairment delays repair so that tissue replacement from progenitor cells becomes the dominant pathway to repopulate regions of injury [20].

\section{Vascular Obstruction in Chronic Liver Disease}

Hepatic vein obstruction is a prominent histologic finding in cirrhosis, whether caused by alcohol, NASH, hepatitis B, or chronic biliary disease $[14 \bullet \bullet, 21 \bullet, 22-26]$. Obstruction is also found in early NASH [25.], alcoholic hepatitis, and in recurrent hepatitis $\mathrm{C}$ after transplantation $[27,28]$. Small portal vein obstruction is found in biopsies with chronic viral hepatitis [26] and in all cirrhotic explant livers [24, 29].

In the present study, high-grade ( $>50 \%)$ obstruction of HVs were found in $15.0 \%$ and $66.5 \%$ of recognizable HVs in mild cirrhosis and severe cirrhosis, respectively. After correction for collapse of tissue, patent HVs were decreased by 59\% in mild cirrhosis and 94\% in severe cirrhosis (Fig. 1a, b).

The involvement of small $(20-100 \mu \mathrm{m})$ and larger HVs was also measured. In stage 4A cirrhosis, high-grade obstruction was found in $63.4 \%$ of small and $26.5 \%$ of larger HVs. Comparable figures in $4 \mathrm{C}$ cirrhosis were $83.9 \%$ and $47.3 \%$. In a study of needle biopsies with NASH, small HVs $(<30 \mu \mathrm{m})$ showed increasing obstruction from stage F0 to F4 [25•]. Together, these data confirm that HV obstruction begins in small $\mathrm{HVs}$ and progresses to involve more HVs and larger HVs as disease advances in severity. The relocation of original hepatic veins to the septa is a
Fig. 3 Congestive injury in early stage disease. a-d. (Chronic HBV). a At low magnification, many mild stress zones are present with minimal atrophy and sinusoidal dilatation. In this field, there are several "healed" lesions (i.e., closed-PELs) seen as delicate septa or cords (boxed lesion enlarged in $\mathbf{c}$ ) and one open-PEL with active congestive necrosis (lesion between arrows, enlarged in $\mathbf{b}$ ). The open-PEL has small hepatic veins at each end (white arrows) connected by obstructed HV segments and the zone of necrosis between. A portal tract (black arrow) is connected to the zone of necrosis by a thin row of dilated sinusoids that joins the PEL at the red arrow. b Enlargement of the open-PEL shows that there is an irregular central cord of collagen that joins the two hepatic veins. This cord appears to be the remains of a segment of necrotic hepatic vein whose lumen has collapsed. The adjacent portal tract connects to the cord at the arrow. (The connection is poorly seen at this tissue level. Similar open-PELs are shown in $\mathbf{h}$, $\mathbf{i}$, and Fig. 4a and similar but closed-PELs are shown in $\mathbf{c}$ ). c There are thin septa or cords linking numerous vascular structures. The large hepatic vein (labeled HV) is closely adherent to a large portal tract (pair of open arrows), indicating a closed-PEL. There is an artery, part of the large portal tract, attached to the large HV (small solid arrow). The 3 large arrows mark arteries, indicating more small portal tracts within this network of PELs. Lesions from this case are also shown in Fig. 4 a-g). d A large hepatic vein has many attached portal tracts, indicating at least 5 separate closed-PELs (arrows on attachment points). e-g (HCV). e There are many small PELs identified by close approximation of portal tracts (PTs) and HVs (arrows indicate PTs in PELs). HVs are decorated with glutamine synthetase (GS) positive perivenular hepatocytes. The box indicates the region shown in $\mathbf{f}$ and $\mathbf{g}$. (GS stain). f. Close approximation of PT (large arrows) with HV (small arrows). (GS stain). g Multiple arterial twigs from the PT (black arrows) are adjacent to the HV wall (white arrows). These likely represent functional A-HV shunts. (CD34 stain). h (NASH). A region studied with 15 serial sections. This field has a large open-PEL with a linear region of hemorrhagic necrosis. The region is partially collapsed, causing approximation of adjacent PTs (black arrows) and hepatic veins (white arrows). The HV has a large obstructed branch (ring of white arrows) and partially destroyed smaller branch (line of white arrows), all within the zone of necrosis. The smaller branch has the same appearance seen in $\mathbf{b}$ and $\mathbf{i}$, caused by HBV and $\mathrm{HCV}$, respectively. The PTs and HVs are distended by edema and hemorrhage making them difficult to identify. Serial sections confirmed identification of some structures. Multiple arterial profiles (open arrows) in the necrotic zone emanate from a PT and represent A-to-HV functional shunts. Portal veins are largely obstructed. This zone of necrosis represents a pressurized common channel (PCC) where many adjacent sinusoids are destroyed (see text for PCC description). i (HCV) An openPEL showing necrosis and hemorrhage adjacent to a necrotic HV wall that is connected to the parent HV (above) and a PT (attached perpendicularly). The collagen fibers in the HV wall are splayed. $\mathbf{j}-\mathbf{l}$ (HCV). A PEL showing PT attachment to an HV. The vein wall is congested and the lumen is partly obstructed. The PT is confirmed by ducts visible on CK7 stain (k) and arteries visible on CD34 stain (I). This PEL may be transitioning from level 3 to level 4. m, n (HCV). Large vessels with intramural edema and hemorrhage ( $\mathrm{HV}$ in $\mathbf{m}, \mathrm{PT}$ in $\mathbf{n}$ ), similar to lesions in smaller vessels in Fig. 4. (All sections are stained with elastic trichrome except as noted above. (Etiology: HBV in a-d, HCV in e-g and i-n, NASH in $\mathbf{h}$, all in Laennec stage F2 with minimal or no activity. Patients with oxaliplatin exposure were excluded.)

useful observation that clarifies the origins of cirrhosis $4 \mathrm{~A}$ (see legend to Fig. 1c).

Major obliteration of hepatic veins explains the prominent congestive features found in cirrhotic livers (Fig. 2). Many regenerative nodules have zones of sinusoidal congestion 

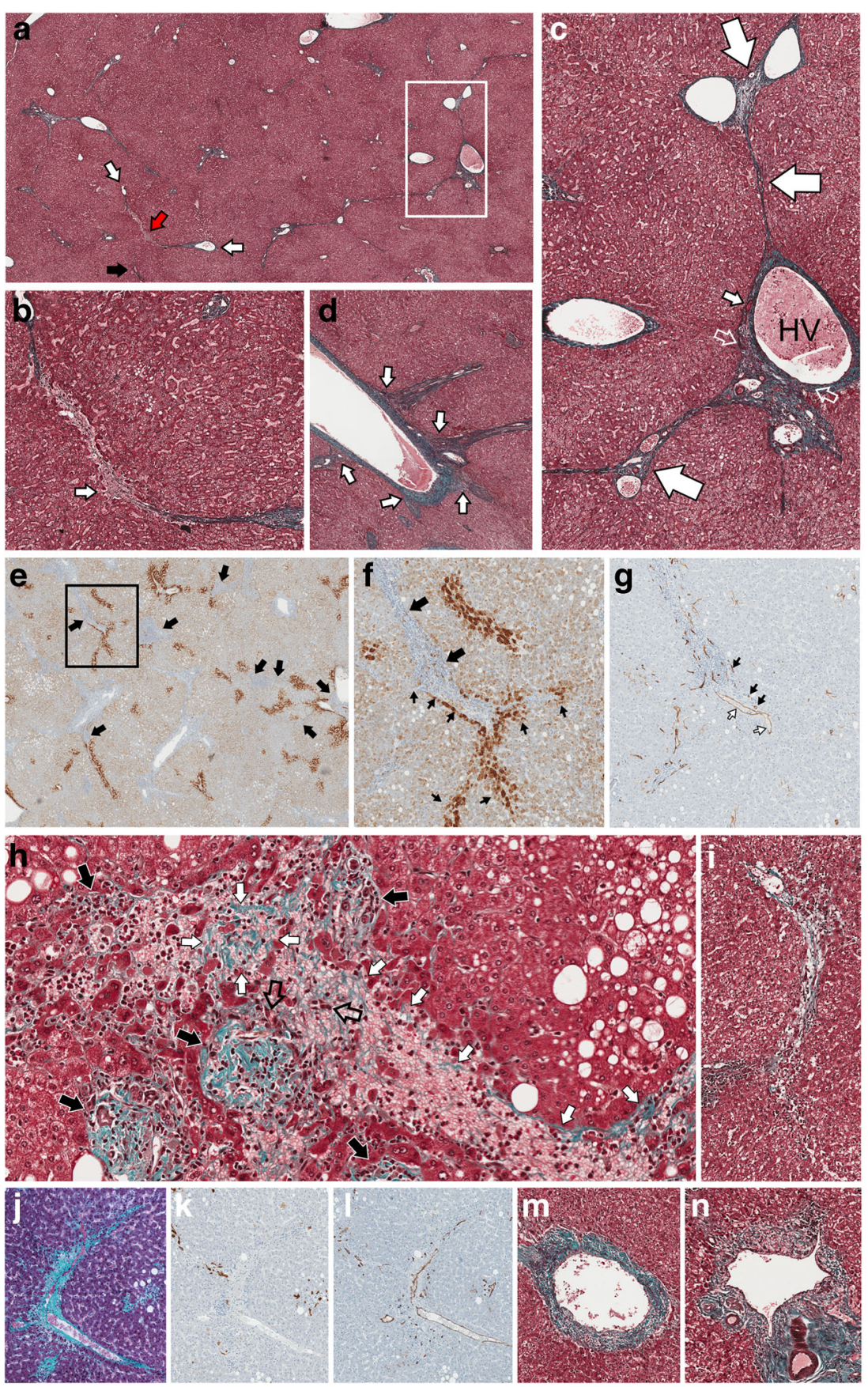

and collapse that lead to subdivision and remodeling of nodules into two or more smaller nodules. The congested zones also contain red blood cells that have dissected into vein walls, causing luminal compromise. These features indicate that congestion is a significant mechanism causing progression from mild to severe cirrhosis. Vascular obstruction in early stage disease is confined to terminal and small branches (Fig. 3b, $\mathrm{e}-1$ ); in cirrhosis, larger vessels are involved (Figs. $2 \mathrm{~h}, \mathrm{j}, \mathrm{k}$ and $3 \mathrm{~m})$. Vascular obstruction is largely confined to PELs (i.e., septal regions) where hepatocytes have been lost. Lesions in small hepatic veins are difficult to identify because vein walls are composed of separate small collagen bundles that are easily obscured by collapse and fibrosis.

\section{The Parenchymal Extinction Sequence}

Parenchymal extinction is a process that evolves with a spectrum of histological appearances (Figs. 2, 3 and 4). These findings were analyzed to create a temporal "parenchymal extinction sequence" that facilitates an understanding of the histologic transitions that eventuate in cirrhosis. Table 1 defines nine levels in the natural history of PELs, with some 

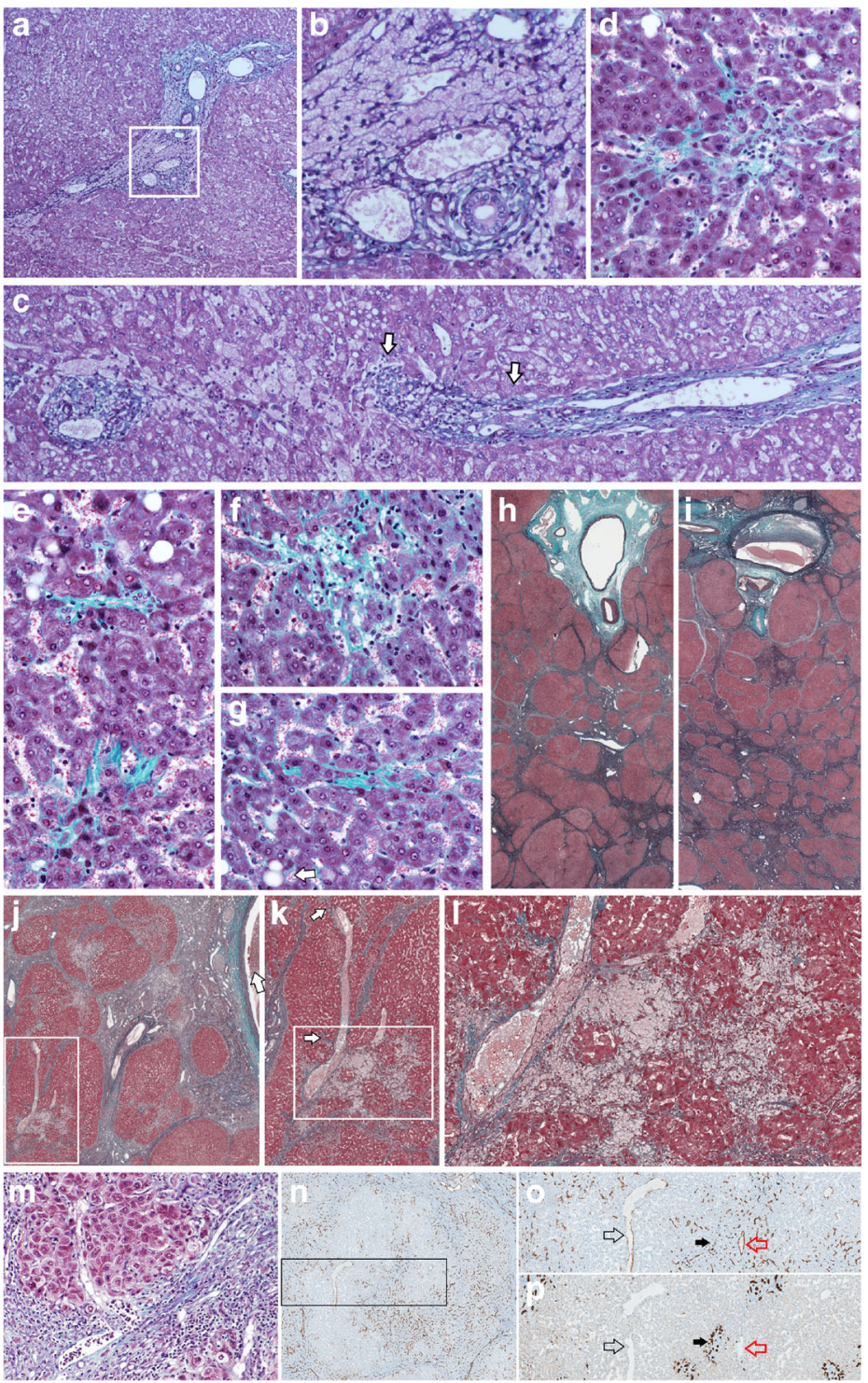

Fig. 4 Lesions showing congestive portal disease, healed vascular remnants, reversed PV flow, and arterialization. a-c Congestive hepatic portopathy in early stage chronic hepatitis B. a This large portal tract has congestion and edema that involves most of the portal tract as well as sinusoids (left). b Closer view shows the portal vein walls and the entire interstitium is suffused with red blood cells. c Congestive involvement is more often seen in smaller portal tracts, as shown here between arrows. There is also parenchymal involvement with sinusoidal dilatation and hepatocellular necrosis. d-g Healed congestive or inflammatory lesions in PTs and HVs (HCV, F2). After healing, PVs are often obstructed or not visible, probably from intimal injury such as shown in $\mathbf{b}$. Portal stroma is also resorbed, leaving only the heaviest fibers that are splayed apart by regenerating hepatocytes. Normal HVs have collagen bundles that vary in size with the diameter of the vein. These bundles are splayed after injury. (PTs in $\mathbf{d}$ and $\mathbf{e}$ top. HVs in $\mathbf{e}$ bottom, $\mathbf{f}$, and $\mathbf{g}$ ). $\mathbf{h}, \mathbf{i}$ Cirrhosis with healed portal vein thrombosis confined to the right lobe. The thrombosed region has more severe cirrhosis, characterized by smaller nodules and wider septa. There was also more congestion on the right (not shown). This could be explained by loss of retrograde venting capacity in the thrombosed lobe. $\mathbf{j}-\mathbf{I}$ PV invaded by hepatocellular carcinoma in mild HBV cirrhosis. $\mathbf{j}$ The tumor is on the right (arrow). Boxes indicate areas enlarged in $\mathbf{k}$ and $\mathbf{l}$. There are severe congestive lesions largely confined to the region near the obstructed PV. This shows the effect of removing the PV as a venting pathway. $\mathrm{k}$. The HV wall is edematous and congested with narrowed lumen at points where PTs (and arterial supply) are attached (arrows) or nearby. $\mathbf{l}$. The portal tracts and sinusoids are largely destroyed by the suffusion of red blood cells, leaving numerous disorganized arteries and ducts in the hemorrhagic region. $\mathbf{m}$ A cirrhotic nodule that drains into a portal vein in the adjacent septum. This is visualization of the well-known phenomenon of reversed flow in the presence of severe HV obstruction. (trichrome stain). $\mathbf{n}-\mathbf{p}$ Severe alcoholic cirrhosis with arterialization of sinusoids (aka capillarization). n A cirrhotic nodule with most arteries in portal tracts outside the nodule. CD34 shows the location of these arteries as well as portal and hepatic vein endothelial cells. Sinusoidal endothelial cells (SEC) are normally CD34-. In this case, approximately half the SEC are CD34+. (Boxed area enlarged in $\mathbf{o}$ and $\mathbf{p}$ ). $\mathbf{o}, \mathbf{p}$ On the left, many sinusoids are dilated, indicating response to pressure and/or ischemia. In these CD34- stress zones, the HV (open arrows) is distant from the arteries outside the nodule. On the right, a CD34+ (arterialized) zone has small caliber sinusoids, possibly because they are protected from arterial pressure by the CD34+ SEC. In this region, the HV (red arrows) is close to the portal tract (black arrows) which is the arterial source. (CK34 in $\mathbf{n}$ and $\mathbf{o}$, CK19 in p). (Patients with oxaliplatin exposure were excluded. All sections stained with elastic trichrome stain, except as noted above.) 
Table 1 Histological sequences

\begin{tabular}{|c|c|c|}
\hline \multirow{2}{*}{$\begin{array}{l}\text { Laennec stage } \\
\text { Stage (description) }\end{array}$} & \multicolumn{2}{|c|}{ The parenchymal extinction sequence (PES) } \\
\hline & Level & Definition (comment) \\
\hline $0-1($ septa-) & 1 & Stress zones with variegation of the color of hepatocyte cytoplasm in clusters the size of lobular units. \\
\hline \multirow[t]{2}{*}{$0-1 /$ septa -$)$} & 2 & Stress zones with hepatocellular atrophy. \\
\hline & 3 & $\begin{array}{l}\text { open-PELs - Small region of hepatocellular dropout - necrosis or apoptosis- without collapse (represents } \\
\text { necrosis, if recent, or a pressurized common channel (PCC) if chronic, occurring after lysis of a number } \\
\text { of adjacent sinusoidal walls) }\end{array}$ \\
\hline $\begin{array}{l}1 \text { (septa-, with portal } \\
\text { expansion) }\end{array}$ & 4 & $\begin{array}{l}\text { closed-PELs - Small region of hepatocellular dropout with collapsed architecture and approximation of } \\
\text { portal tracts and hepatic veins. (Lesions are usually detectable with glutamine synthetase stain.) }\end{array}$ \\
\hline 2 & 5 & $\begin{array}{l}\text { Merged PELs that form small aggregates or short septa containing 2-3 PTs. (These aggregated PELs may } \\
\text { also be open or closed.) }\end{array}$ \\
\hline $3-4 \mathrm{~A}($ septa++) & 6 & Extended PEL aggregates that form a network of septa (thin septa if closed). \\
\hline 4B-4C (septa+++) & 7 & $\begin{array}{l}\text { Large PEL aggregates containing many portal tracts crowded together, with each PT surrounded by } \\
\text { collapsed stroma rather than hepatocyte plates }\end{array}$ \\
\hline $\begin{array}{l}\text { Regressing cirrhosis (septa } \\
\text { PES level 8) }\end{array}$ & 8 & $\begin{array}{l}\text { Septa are thin links between remnants of portal tracts and hepatic veins (found in early regression of } \\
\text { septa/cirrhosis) }\end{array}$ \\
\hline $\begin{array}{l}\text { Regressing cirrhosis (septa } \\
\text { PES level 9) }\end{array}$ & 9 & $\begin{array}{l}\text { Former septa that are fragmented, leaving collagenous tags attached to recognizable PT remnants or HV } \\
\text { remnants but not definitely connected to a septum (found in late regression of septa/cirrhosis. Some } \\
\text { of these tags may be residual strings of PT stroma or linear remnants of HVs walls.) }\end{array}$ \\
\hline
\end{tabular}

The development of parenchymal extinction lesions demonstrate a spectrum of findings that can be divided into 9 histologically identifiable levels. Early changes (level 1-5) were more prevalent in stage 1-2 disease but could be seen in cirrhotic livers. Levels 6-9 were mostly seen in stage 4 disease. Remodeling during regression causes septa to become perforated membranes or thin cords, scored as PES level 8 or 9

highlights described here. Because individual PELs develop over a prolonged period of time, a mixture of early, late, and regressed lesions can usually be seen in the same specimen.

Stress Zones (Level 1 and 2 Lesions) These lesions are the precursors that may evolve into PELs (Figs. 2c-e and 3a-d). In early-stage disease, stress zones are subtle, caused by a mild degree of vascular derangement, described below. In cirrhosis, when most PELs have already aggregated into a network of septa (as level 5-7 PELs), there continue to be stress zones, as well as level 3 and 4 lesions, indicating ongoing congestive stress in the regenerative nodules.

In early-stage disease, stress zones and level 3 lesions may be initiated by the primary disease (generally accompanied by inflammation) but if inflammation is minimal or absent, the lesions likely progress because of added congestive features, including endothelial leak, fluid translocation, and vascular compression. In established cirrhosis, level 2-3 lesions often have no evidence of inflammation, suggesting these lesions are entirely congestive in nature with little dependence on primary disease activity.

Stress zones may be explained as a response to flow changes induced by the earliest obstructive lesions in portal and hepatic veins, some of which are accompanied by small shunts and reactive hyperemia. Shunts would cause local regions with higher outflow pressure (IOI, defined below), leading to sluggish flow, dilatation, as well as atrophy in level 2 lesions. Glycogen depletion may be caused by local diversion of insulin delivery.
Level 3 and 4 Lesions (Open- and Closed-PELs) Open-PELs (level 3) are those in which the hepatocytes are lost and SEC are lifted or lost, but the residual tissue space contains blood because the lesion has not fully collapsed (Figs. 2f, g, 3b, h, i, and $4 \mathrm{c}, \mathrm{k}, 1)$. Closed-PELs (level 4) have collapsed, so that the area of tissue loss may be inapparent except for approximation of adjacent portal tracts and hepatic veins (Fig. 3c-g, j-l). Open-PELs may indicate recent necrosis, but may also be seen in late regressed disease, suggesting that they are open to afferent flow but have significant outflow obstruction.

Small level 3 PELs are often linear, distributed as a thin sleeve of necrotic hepatocytes surrounding a collapsed HV wall, and connecting to congested portal tracts that have splayed stromal fibers (Figs. 3h, n, and 4b). After the lesions have collapsed, the fibers of HV walls and PT stroma often remain splayed (see Fig. 4d-g). In level 4 PELs, the points of attachment of PT and HV are often arranged perpendicularly (Fig. 3a, b, i-1).

Both open- and closed-PELs often show arteries adjacent to the hepatic venule (Fig. 3c, g, h, 1) or inside a hepatic vein lumen, representing an A-HV shunt. Shunts would allow local arterial flow to drain, thereby lowering intra-lesional tissue pressure. The recognition that there is an apparent evolution of stress zones, to open-PELs to closed-PELs allows creation of a coherent pathogenic model, as illustrated in Figs. 5 and 6.

PELs are constantly healing, especially after congested areas drain, presumably through A-HV shunts. Thus, in pre-cirrhotic and early cirrhotic chronic liver disease (F3/4A), most PELs are 


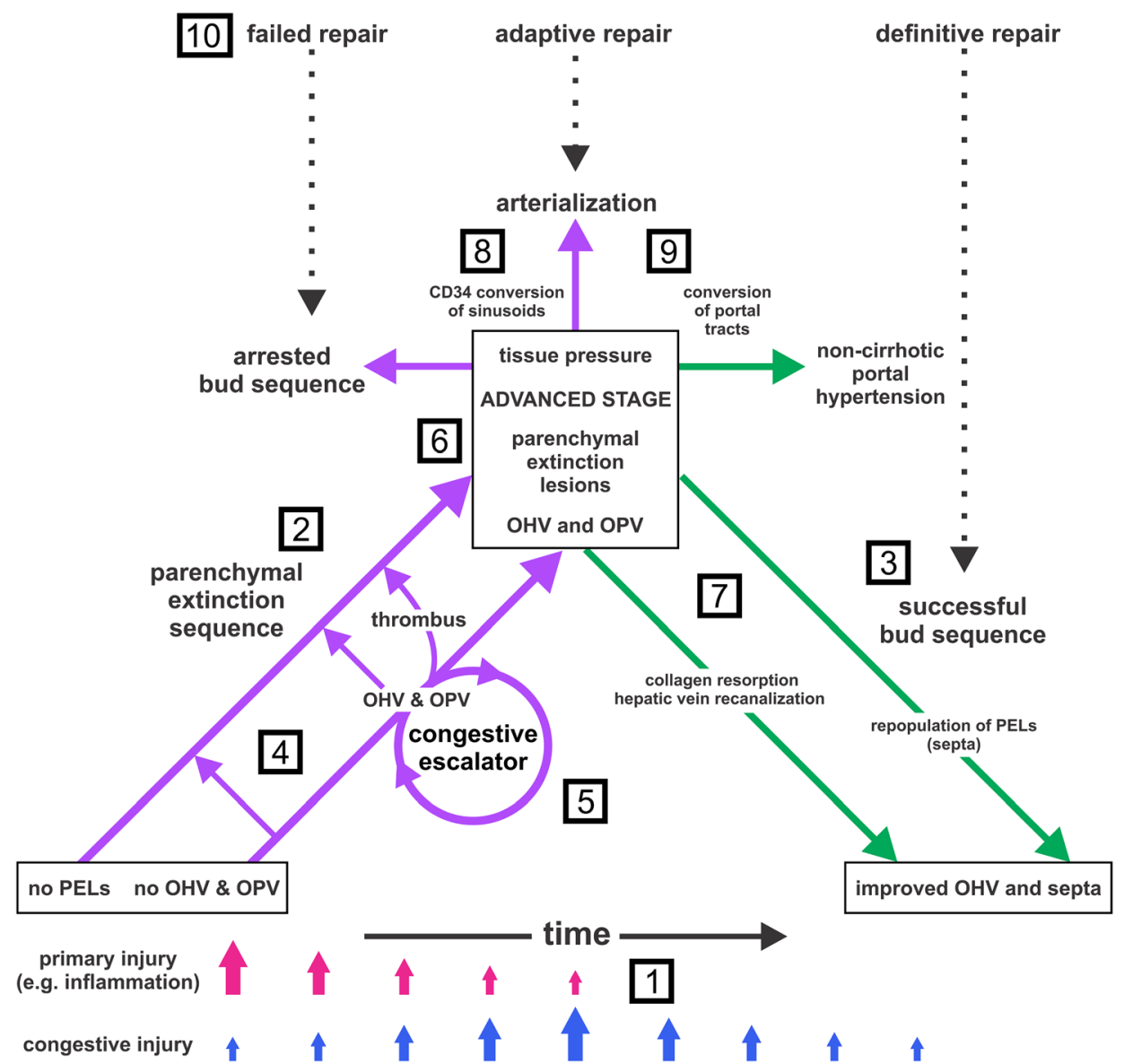

Fig. 5 Diagram to summarize the vascular changes and congestive escalator in the pathogenesis and regression of cirrhosis. The following explanatory notes refer to the numbered boxes in the diagram. (1) There are 2 main injury types. The primary injury depends on the specific disease. Most diseases cause inflammation that damages hepatocytes, less often mainly the vessels, and rarely primary thrombotic change. Secondary congestive injury is a response to vascular damage occurring in the course of the primary injury, but also in response to congestioninduced vascular damage. Severity of the injury types varies with time, depending on activity of the primary disease and therapy. Congestive injury is dominant in severe cirrhosis after primary injury has remitted. Once there is severe obstruction of hepatic veins, the congestive escalator continues to cause progressive vascular and hepatocellular injury even if primary inflammatory factors have abated. OHV, OPV obstruction of hepatic and portal veins. $(2,3)$ Two histologic sequences can be recognized. The parenchymal extinction sequence tracks progression, as detailed in Table 1. The bud sequence tracks regeneration from progenitor cells. These are indicated by ascending purple arrows and descending green arrows, respectively. The lines are double to indicate parallel processes. (4) The progression lines (purple) indicate the lesions involved in tissue destruction. OHV and OPV (right line) worsen (involving more and larger veins) to cause PELs to increase in number and size (left line). Factors causing progression are those that cause increased tissue pressure. These include active primary disease (usually inflammation), activation of the congestive escalator, and secondary thrombosis. Vascular obstruction and the resulting parenchymal extinction sequence are the most important events leading to progression of disease. Factors favoring regression include those that lower tissue pressure. These include cessation of inflammation, relief from congestion (reduction of hyperemia, recanalization, and generation of new drainage channels, especially sinusoids and veins). (5) The circle indicates a positive feedback loop called "congestive escalator." This

operates because vascular obstruction causes more congestion that causes more vascular obstruction, etc. This cycle explains why OHV and OPV lesions increase in number and progress to involve larger vessels. It also explains how disease can progress even after the primary disease has remitted. Initiation of the congestive escalator is explained in Fig. 6a-e. (6) Advanced stage, usually called cirrhosis, can be measured by many quantitative parameters. Three are selected here but others include collagen concentration and portal pressure. (7) Advanced liver disease can regress through regeneration (esp. the bud sequence) and resorption of collagen. The former repopulates regions of extinction by replacing the lost hepatocytes and sinusoids. Collagen resorption facilitates recanalization of obstructed veins and a decline in tissue pressure and congestion. $(8,9)$ Arterialization may be considered as two types of adaptive repair that occur in response to elevated pressure. Arterialization includes conversion of sinusoidal endothelial cells to express CD34 (aka capillarization) [30] and conversion of the portal tracts, by obstruction of PVs, to supply only arterial blood. These are called "conversion," because once modified for arterial inflow, recovery to normal structure is unlikely to occur [31]. Conversion of portal tracts is largely completed by the time moderate-severe cirrhosis has occurred. After regression of cirrhosis, converted portal tracts (and OPV) persist and may manifest as non-cirrhotic portal hypertension [32]. (10) Repair processes do not always return tissues to their normal state. The bud sequence can provide a definitive repair with repopulation of PELs [20]. However, the bud sequence becomes arrested in highly congested tissue, leading to persistent septa that represent failed repair [33]. Arterialization may protect sinusoids against arterial pressure, but this is an adaptive repair with less than optimal function. Similarly, portal tract conversion with persistent OPV contributes to portal hypertension in cirrhosis and in non-cirrhotic portal hypertension. These features are illustrated in a video (see Fig. 7) 
level 4-6. In F4B/4C, PELs are mostly level 7 aggregates seen as numerous and broad septa. As these later stages regress and aggregated PELs become separate, the PELs become more delicate and resemble those seen in F3/4A. The distinction can be made, if sufficient tissue is available, by the larger number of aggregated PELs (septa) in regressing cirrhosis, often with curved contours. In F3/4A, there are likely to be some portal tracts that are not linked. There will be overlap, so a previous biopsy with cirrhosis may be needed for confirmation.

\section{Pressurized Common Channels (PCC) as Shunts that Transmit} Tissue Pressure Aggregated PELs may also be open or closed. Large aggregates of open-PELs are seen as congested septa. Congested septa can be understood as "pressurized common channels" (PCC) that formed when sinusoidal walls were destroyed and then incorporated into the septum. PCCs are interconnecting shunts that may be fed by arteries from many portal tracts throughout the septum, with drainage into available hepatic or portal veins. These channels can transmit elevated tissue pressure, potentially throughout large portions of the liver. This is the mechanism whereby the adventitia of large hepatic veins is exposed to high pressure, leading to congestive injury and luminal narrowing (Fig. 2k).

Regression of PELs Level 7-8 PELs show regressed features. These lesions are invariably collapsed (closed) suggesting that any contained channels are not pressurized. Tissue pressure is likely to be low, consistent with successful outflow re-vascularization, and healthy parenchymal regeneration.

\section{Congestive Vasculopathy and the Congestive Escalator}

Function and survival of liver parenchyma require that flow and pressure remain within a physiological range. In the course of liver disease, there is vascular obstruction (resistance to flow) and local hyperemia that causes a departure from this range, summarized as "in-out-imbalance"(IOI). Congestive injury occurs in a vascular system when there is IOI, that is, when inflow (expressed as hepatic artery flow, HAF) exceeds the outflow capacity of the system (expressed as hepatic outflow capacity, HOC). HAF is taken as an approximation of the inflow load on the system, since portal vein flow is probably insignificant after early obstruction of small portal veins has occurred, mostly in stages F2-3. HOC can be defined as the maximum flow that can be carried without causing injury to the vascular bed.

In-out-imbalance provides a conceptual framework to summarize the causes and pathogenesis of congestive injury and the congestive escalator. Thus, HAF is increased by reactive hyperemia (in response to inflammation, ischemia, portal vein obstruction, or shunting). HOC is decreased by obstruction of hepatic veins or portal veins (by intimal thickening or external compression). In-out-imbalance causes an elevated transmural pressure gradient that causes endothelial injury, vascular leak, with transudation and edema. Expansion of tissue compresses adjacent tissues including veins (compartment syndrome effects), leading to more increase in IOI and more vascular injury. Thus, we can summarize the sequence of events: IOI causes congestive vasculopathy which leads to parenchymal extinction and cirrhosis (Table 1, Figs. 5 and 6). In overview, the physiological state of in-out-imbalance can explain, in large measure, the progression of chronic liver disease. A video of these events is available (Fig. 7).

\section{Discussion}

The Vascular Hypothesis Summary Vascular obstruction, particularly involving sinusoids and hepatic veins, causes tissue congestion which itself induces further vascular obstruction leading to focal necrosis involving hepatocytes and sinusoidal endothelial cells, in a process called parenchymal extinction.

The PE Sequence Parenchymal extinction proceeds through a sequence of tissue changes beginning with stress zones that progress to atrophy, necrosis, collapse, and ultimately, regression of PELs and septa. Parenchymal extinction progresses by increase in number and size of PELs until aggregated PELs form a continuous web of septa typical of cirrhosis. Regression involves repopulation of septa with progenitorderived hepatocytes, detachment of adhesions, and resorption of residual collagen. The forces that drive the lesions are summarized above and illustrated in Fig. 6.

Value of the PE Sequence Model PELs are larger than the minute vascular lesions that cause them. Therefore, PELs are easily visible histologically, especially with glutamine synthetase staining, making them a useful surrogate target for quantitative analysis of microvascular obliteration. PEL size correlates with the size of obstructed hepatic veins, so that sites of obstruction can be identified quickly at low magnification.

The levels within the PE sequence allow one to understand the process of tissue destruction from onset, to aggregation into septa, and regression towards normal. Examination of these transitions allows insight into the timing and quantity of collagen deposition and resorption, and to distinguish these parameters from increase in collagen concentration caused by simple collapse. The open and closed variants allow further evaluation of progressive and regressive states of the vasculature. Areas of parenchymal extinction may repopulate with new tissue that can be localized by EpCAM, CK7, and CK19 distribution. These stains allow evaluation of location and pace of regeneration in relation to regression of cirrhosis.

Laennec stages correlate with features of the PE sequence, potentially allowing new features to be incorporated into a revised Laennec system (Table 1). These would improve the precision of staging in early chronic liver disease. For example, stages $0-4 \mathrm{~A}$ 


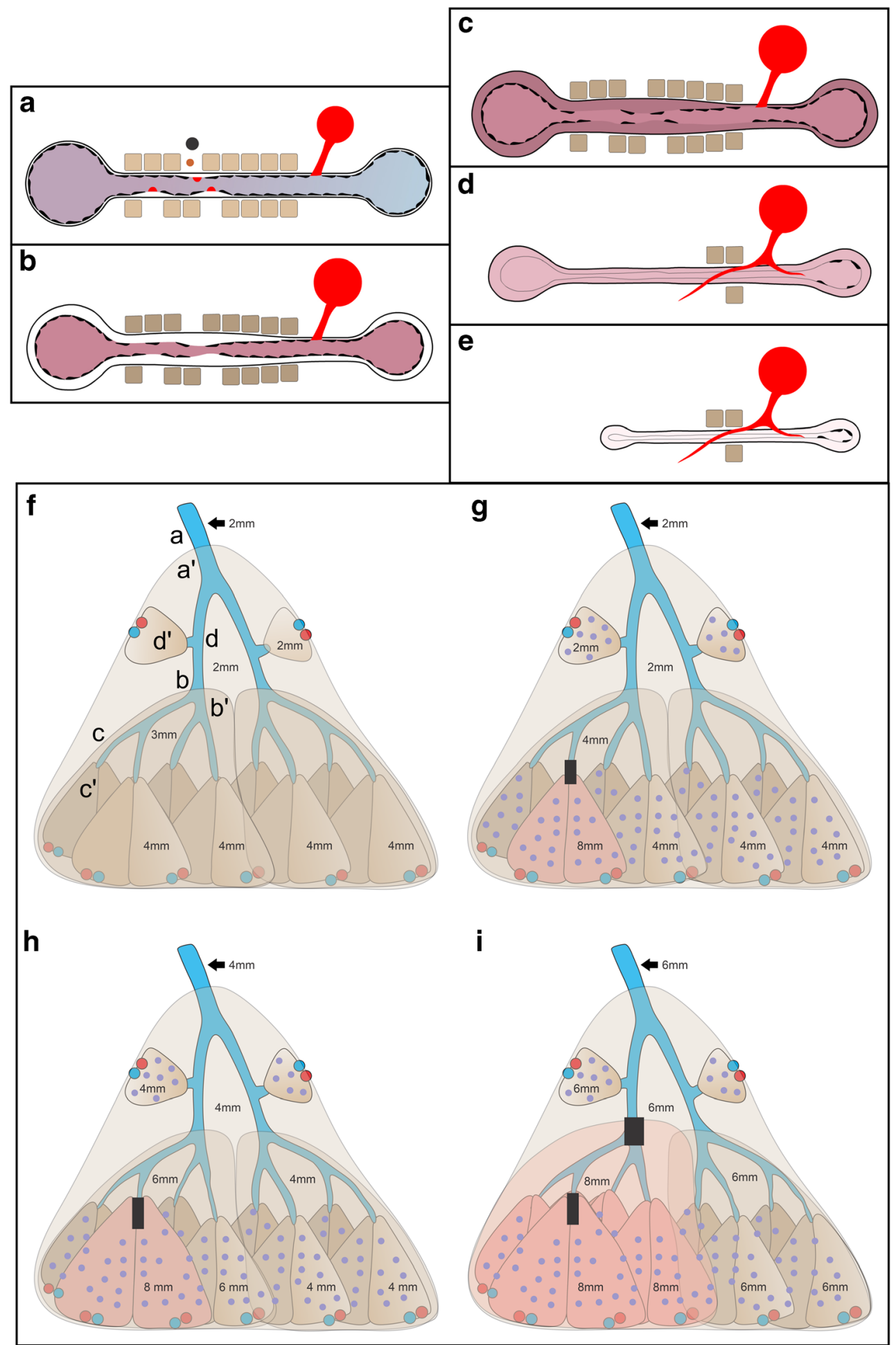

can be defined quantitatively by the percentage of portal tracts that are attached to adjacent hepatic veins by PELs (the VPA index). This exercise is facilitated with the glutamine synthetase stain.

Difficulty Identifying Destroyed Tissue Elements The early events in chronic liver disease have been difficult to characterize, as we and others [34] have found, largely because the microvasculature is usually destroyed beyond recognition. Three approaches have allowed progress in this field. By recognizing that open-PELs are the precursor of closed-PELs, the small hepatic veins could be located and studied at level 3 before tissue collapse that masks the venous damage (Fig. $3 \mathrm{~b}, \mathrm{~h}, \mathrm{i})$. A second approach was to examine level 3 and 4 lesions, with the help of the glutamine synthetase stain, to 
4 Fig. 6 Schematic illustrations of the pathogenesis of early PELs (a-e) and the role of the "nested cone" architecture of the hepatic venous outflow tract in the human (f-i). a The earliest PELs occur at the sinusoidal level. For simplicity, this drawing shows the parenchyma represented by a single sinusoid joining a portal vein (right) and hepatic vein (left), with an artery entering the proximal end of the sinusoid. In chronic hepatitis, PELs are initiated by injury that is directed at hepatocytes but also involves adjacent sinusoidal endothelial cells (SEC). The black dot is a lymphocyte attacking an hepatocyte that is responding with apoptosis. The underlying SEC has rounded up, with widening of the intercellular junctions. On the opposite side of the sinusoid, two SEC have been lifted by subendothelial edema at sites of previous injury. This is evidence of transudation of fluid at sites of leakiness. Focal edema has caused narrowing of the lumen. b The artery has enlarged, and luminal blood has become oxygenated with arterial blood. Several SEC have desquamated. Subendothelial spaces are expanded with edema fluid causing marked narrowing of the lumina of portal and hepatic vein as well as the sinusoid. $\mathbf{c}$ More SEC have desquamated and there is now evidence of hemorrhage with red cells in the subendothelial spaces. d Most hepatocytes are gone but edema and hemorrhage remain. An arterial branch has grown into the sinusoid and is draining into portal vein and adjacent interstitium. This effectively vents the arterial flow away from the damaged sinusoidal lumen, allowing the sinusoid to decompress and collapse. This is an open-PEL (level-3 PEL). e With decompression, there is complete collapse and approximation of the HV and PV. This is a closed-PEL (level-4 PEL). In summary, local inflammation, along with obstruction, is followed by reactive hyperemia that raises sinusoidal pressure that causes more transudation into the space of Disse, venous subendothelial space, and other interstitial spaces. As these spaces become engorged, further obstruction ensues with a positive feedback loop until distension and ischemia lead to SEC sloughing and further hepatocellular necrosis. When many adjacent sinusoids and hepatocytes are involved, a PEL forms, usually with collapse and obliteration of small portal and hepatic veins. Veins become obstructed by mural edema as well as external compression (compartment syndrome effect). This early sequence, as well as progression to severe cirrhosis, are demonstrated in a video available online (see Fig. 7). f The nested cone model in normal human liver. The axial hepatic veins branch dichotomously 4-6 times at acute angles ( 3 shown here: a,b,c). Many small branches arise at right angles (d). Each hepatic vein branch drains a cone of parenchyma that is nested inside larger cones drained by larger branches. The cones are labeled a'-to- $\mathrm{d}^{\prime}$, corresponding to the branches labeled a-to-d. As collateral pathways are few, each cone has its own pressure environment modified by local venous obstruction and hyperemia, as indicated by hypothetical examples, in $\mathrm{mmHg}$. Distal branches have a longer path to the vena cava than side branches of larger axial branches, as described by Mall [35]. The shorter path would likely be associated with lower venous pressure. Obstruction is due to luminal reduction as well as external compression. The nested cone architecture facilitates the migration of external compression to involve larger vessels as adjacent tissue expands. $\mathbf{g}$ In chronic hepatitis, there is widespread mild to moderate inflammation (denoted by dots) leading to local sinusoidal and venous obstruction, as described in panels a-e. One cone has become more congested than others as its hepatic vein is compromised, with a local rise in pressure. h Hyperemia and vascular leak has caused expansion of the affected cone that now is compressing adjacent cones, spreading the pressure rise to them. $\mathbf{i}$ Vascular obstruction has extended to a larger vein causing 3 more small cones to expand with more compression of the cones on the right as well as higher in the tree

show in detail small PELs, small hepatic veins, and points of venous adhesion to portal tracts (Fig. 3e-g). A third approach was to examine early microvascular lesions in more acute diseases, where damaged terminal hepatic venules were easier to identify before tissue collapse and fibrosis.
Mechanism of Fibrosis (Inflammatory Vs Congestive) Edema is an important clue that expansion of interstitial- and intravascular spaces contributes to luminal obstruction and the formation of pressure gradients [36]. Congestive fibrosis occurs at sites where translocation of fluid and blood components occurs. This is seen in the skin of the lower legs in patients with chronic heart failure, where "brawny edema" reflects the development of subcutaneous fibrosis. Fibrous capsular thickening of the liver and spleen is also seen in such patients and in cirrhosis [37, 38]. Exudation generates deposits of relatively static protein-rich fluid that activates fibroblasts as part of a granulation tissue response (Fig. $2 \mathrm{j}-1$ ).

Mueller hypothesized that elevated sinusoidal pressure is a major factor in the synthesis of collagen in chronic liver disease. He proposed that collagen synthesis was caused by stretching of the hepatic stellate cells and activation of biomechanical signaling. This hypothesis was based on correlation with liver stiffness and pressure but without discussion of vascular lesions and other histologic evidence [39].

Role of Fibrosis in the Pathogenesis of Cirrhosis Fibrosis occurs at many sites in the cirrhotic liver. Fibrosis within vascular channels would be the most potent to increase congestion and progression of parenchymal extinction. However, increased tissue stiffness caused by collagen deposition at any site would impair dilatation of the micro-vasculature in response to hyperemia. With this confinement, SEC injury, transudation, and parenchymal extinction would occur with lesser degrees of hyperemia.

Primary Injury Is Necessary to Drive Progression of Disease,
Until a Tipping Point Is Reached The severity of primary injury determines the clinical outcome in chronic disease. When primary disease is suppressed by spontaneous remission or therapy, most livers with mild to moderate cirrhosis regress substantially. In this population, the primary injury is required to initiate or maintain progression. Patients with severe cirrhosis may not regress, even with remission, and these patients (enriched in the transplant population) may have reached the tipping point where obstructed veins and destroyed sinusoids are sufficient to support progression (i.e., drive the congestive escalator). New onset of tense ascites, portal vein thrombosis, anemia and hypoxemia, and super-infection are factors that may tip the balance towards a congestive progression.

Histologic features of active viral disease or steatohepatitis, adjacent to PELs, suggest that a primary inflammatory mechanism may still be active in PEL formation. The presence of open-PELs or congested or edematous septa but without active inflammation suggests that the tipping point has been reached. 
Role of Thrombus in Progression of Chronic Liver Disease Thrombus may be a factor in progression of disease, as seen in Budd-Chiari syndrome and chronic congestive heart failure. In chronic viral and alcoholic disease, histological thrombosis of small hepatic and portal veins does occur but is rarely identified, in part because recent thrombus is rapidly replaced by less specific fibrous plugs. Sub-microscopic fibrin deposition could also have an effect. Detection of large portal vein thrombi, often achieved by clinical imaging, has been a major focus of attention. Patients with cirrhosis who received enoxaparin, had decreased rates portal vein thrombosis as well as less decompensation on follow-up [8••]. Portal vein thrombosis may be an important cause of decompensation. In severe cirrhosis, portal vein flow is often retrograde, indicating the importance of the portal vein as an outflow tract (see Fig. 4h, i).

\section{Role of Endothelial Cell Injury}

The vascular model presented here is focused on the potential of SEC injury to initiate chronic liver disease. This is supported by finding SEC injury in most primary diseases that can progress to cirrhosis. It is not surprising that hepatitis $\mathrm{C}$ is synergistic with toxic agents used in preparation for bone marrow transplantation [40] in causing liver decompensation. The same agents used alone can cause hepatic SOS/VOD. At doses of oxaliplatin used for colon carcinoma therapy, patients with advanced cirrhosis are at increased risk for hepatic decompensation [41].

The vascular model allows the rationale for various therapies to be understood in anatomic terms. Thus, any procedure that lowers intrahepatic pressure or outflow resistance might be of benefit. Simvastatin has hepatoprotective properties by several mechanisms, including protection of SEC [42, 43•].

\section{Conclusion}

The vascular model described here, provides an anatomic framework to understand the mechanisms that drive progression of chronic liver disease in the human.

- Cirrhosis is the morphologic result of parenchymal extinction that leads to collapse of tissue to form septa. Parenchymal extinction is characterized by focal loss of hepatocytes as well as destruction of sinusoids and obstruction of small portal and hepatic veins.

- Parenchymal extinction is initiated by a primary liver disease that causes vascular injury, usually at the level of sinusoidal endothelial cells. Sinusoidal injury progresses to parenchymal extinction by a sequence of events that involve vascular leak, transudation into vein walls and interstitium, ischemia, and hyperemia. The resultant microvascular obstruction causes further congestive injury and the extension of venous obstruction to larger vessels in a positive feedback loop called the "congestive escalator,"

- The human liver is particularly vulnerable to this congestive escalator because the "nested cone" architecture, mandated by the long and branched hepatic vein tree, is susceptible to "compartment syndrome" effects. Animals used for most experimental models have a very short hepatic venous tree that is unlikely to produce the prominent congestive effects found in human liver disease. This difference suggests that the pathogenesis of cirrhosis in humans and animal models may be quite different.

\section{Compliance with Ethical Standards}

Conflict of Interest The author declares he has no conflict of interest.

Human and Animal Rights and Informed Consent This article contains human data approved by the Nova Scotia Health Authority Research Ethics Board, No. 1007257.

Open Access This article is licensed under a Creative Commons Attribution 4.0 International License, which permits use, sharing, adaptation, distribution and reproduction in any medium or format, as long as you give appropriate credit to the original author(s) and the source, provide a link to the Creative Commons licence, and indicate if changes were made. The images or other third party material in this article are included in the article's Creative Commons licence, unless indicated otherwise in a credit line to the material. If material is not included in the article's Creative Commons licence and your intended use is not permitted by statutory regulation or exceeds the permitted use, you will need to obtain permission directly from the copyright holder. To view a copy of this licence, visit http://creativecommons.org/licenses/by/4.0/.

\section{References}

Papers of particular interest, published recently, have been highlighted as:

- Of importance

- Of major importance

1. Garcia-Pagan JC, Gracia-Sancho J, Bosch J. Functional aspects on the pathophysiology of portal hypertension in cirrhosis. J Hepatol. 2012;57(2):458-61.

2. Peverill W, Powell LW, Skoien R. Evolving concepts in the pathogenesis of NASH: beyond steatosis and inflammation. Int J Mol Sci. 2014;15(5):8591-638.

3. Trautwein C, Friedman SL, Schuppan D, Pinzani M. Hepatic fibrosis: concept to treatment. J Hepatol. 2015;62(1 Suppl):S15-24.

4. Seki E, Brenner DA. Recent advancement of molecular mechanisms of liver fibrosis. J Hepatobiliary Pancreat Sci. 2015;22(7): $512-8$. 
5. Karin D, Koyama Y, Brenner D, Kisseleva T. The characteristics of activated portal fibroblasts/myofibroblasts in liver fibrosis. Differentiation. 2016;92(3):84-92.

6. Koyama Y, Brenner DA. Liver inflammation and fibrosis. J Clin Invest. 2017;127(1):55-64.

7. Yang L, Kwon J, Popov Y, Gajdos GB, Ordog T, Brekken RA, et al. Vascular endothelial growth factor promotes fibrosis resolution and repair in mice. Gastroenterology. 2014;146(5):1339-50 e1.

8.• Villa E, Camma C, Marietta M, Luongo M, Critelli R, Colopi S, et al. Enoxaparin prevents portal vein thrombosis and liver decompensation in patients with advanced cirrhosis. Gastroenterology. 2012;143(5):1253-1260 e4. Clinical trial indicating that modulation of coagulation pathways may influence the natural history of cirrhosis.

9. Schepis F, Villa E. Thrombophilic genetic risk factors for liver fibrosis: to screen or not to screen? J Hepatol. 2015;63(6):1311-3.

10.• Tripathi DM, Vilaseca M, Lafoz E, Garcia-Caldero H, Viegas Haute G, Fernandez-Iglesias A, et al. Simvastatin prevents progression of acute on chronic liver failure in rats with cirrhosis and portal hypertension. Gastroenterology. 2018;155(5):1564-77. Animal model indicating that simvastatin may influence the natural history of cirrhosis.

11. Meireles CZ, Pasarin M, Lozano JJ, Garcia-Caldero H, GraciaSancho J, Garcia-Pagan JC, et al. Simvastatin attenuates liver injury in rodents with biliary cirrhosis submitted to hemorrhage/resuscitation. Shock. 2017;47(3):370-7.

12. Turco L, Villanueva C, La Mura V, Garcia-Pagan JC, Reiberger T, Genesca J, et al. Lowering portal pressure improves outcomes of patients with cirrhosis, with or without ascites: a meta-analysis. Clin Gastroenterol Hepatol. 2019.

13. Wanless IR. An hypothesis for the pathogenesis of cirrhosis based on vascular pathology of liver in pre-cirrhotic states. Mod Pathol. 1994; 7:136A.

14.• Wanless IR, Wong F, Blendis LM, Greig P, Heathcote EJ, Levy G. Hepatic and portal vein thrombosis in cirrhosis: possible role in development of parenchymal extinction and portal hypertension. Hepatology. 1995;21(5):1238-47. First hypothesis that vascular obstruction, possibly related to thrombosis, has a role in tissue loss and fibrosis found in human chronic liver disease.

15. Wanless IR. Thrombosis and phlebitis in the pathogenesis of portal hypertension and cirrhosis: the 2-hit hypothesis for the pathogenesis of chronic liver disease. In: Arroyo V, Bosch J, Bruguera M, Rodes $\mathrm{J}$, editors. Therapy in liver diseases. 3rd ed. Barcelona: Masson; 1997. p. 47-50.

16. Wanless IR, Huang W-Y. Vascular disorders. In: Burt AD, Portmann BC, Ferrell L, editors. MacSween's pathology of the liver. 6th ed. Edinburgh: Churchill-Livingstone-Elsevier; 2012. p. 601-43.

17. Edwards L, Wanless IR. Mechanisms of liver involvement in systemic disease. Best Pract Res Clin Gastroenterol. 2013;27(4):471-83.

18. Wanless IR. Physioanatomic considerations. In: Schiff ER, Maddrey WC, Reddy KR, editors. Schiff's diseases of the liver. 12th ed. Oxford: John Wiley and Sons; 2018. p. 73-102. Recent summary of the role of hepatic vascular disease in the pathogenesis and natural history of chronic liver disease.

19. Wanless I. Pathology of Budd-Chiari syndrome and Hepatic vein obstruction. In: Qi X, editor. Budd Chiari Syndrome. Berlin: Springer; 2019. p. in press. Springer Nature Singapore; 2020, 2738 .

20. Stueck AE, Wanless IR. Hepatocyte buds derived from progenitor cells repopulate regions of parenchymal extinction in human cirrhosis. Hepatology. 2015;61(5):1696-707.

21. Goodman ZD, Ishak KG. Occlusive venous lesions in alcoholic liver disease: a study of 200 cases. Gastroenterology. 1982;83: 786-96. Early report of hepatic vascular disease in patients with cirrhosis.
22. Nakanuma Y, Ohta G, Doishita K. Quantitation and serial section observations of focal veno-occlusive lesions of hepatic veins in liver cirrhosis. Virchows Arch (Pathol Anat). 1985;405:429-38.

23. Burt AD, MacSween RNM. Hepatic vein lesions in alcoholic liver disease: retrospective biopsy and necropsy study. J Clin Pathol. 1986;39(1):63-7.

24. Wanless IR, Nakashima E, Sherman M. Regression of human cirrhosis: morphologic features and the genesis of incomplete septal cirrhosis. Arch Pathol Lab Med. 2000;124:1599-607.

25. Wanless IR, Shiota K. The pathogenesis of nonalcoholic steatohepatitis and other fatty liver diseases: a four-step model including the role of lipid release and hepatic venular obstruction in the progression to cirrhosis. Semin Liver Dis. 2004;24(1):99 106. This paper documents hepatic vein obstruction in early NASH. A hypothesis is presented that integrates the roles of elevated insulin levels, lipid toxicity, and vascular obstruction in the pathogenesis of NASH.

26. Guido M, Sarcognato S, Russo FP, Cardin R, Piciocchi M, Colloredo G, et al. Focus on histological abnormalities of intrahepatic vasculature in chronic viral hepatitis. Liver Int. 2018;38(10):1770-6.

27. Malkan G, Humar A, Lilly L, Greig PD, Grant D, Wanless IW, et al. Natural history of recurrent hepatitis C after liver transplantation. Transplant Proc. 2001;33(1-2):1468.

28. Wanless IR, Kutami R, Shiota K. Pathology of recurrent hepatitis C after liver transplantation: a model system to study the histogenesis of cirrhosis. Hepatology. 2001;34(4 part 2):218A.

29. Krasinskas AM, Goldsmith JD, Burke A, Furth EE. Abnormal intrahepatic portal vasculature in native and allograft liver biopsies: a comparative analysis. Am J Surg Pathol. 2005;29(10):1382-8.

30. Arnason T, Fleming K, Wanless IR. Peritumoural hyperplasia of the liver: a response to portal vein invasion by hypervascular neoplasms. Histopathology. 2013;62:458-64.

31. Ward NL, Haninec AL, Van Slyke P, Sled JG, Sturk C, Henkelman RM, et al. Angiopoietin-1 causes reversible degradation of the portal microcirculation in mice: implications for treatment of liver disease. Am J Pathol. 2004;165(3):889-99.

32. Nakashima E, Kage M, Wanless IR. Idiopathic portal hypertension: histologic evidence that some cases may be regressed cirrhosis with portal vein thrombosis. Hepatology 1999;30:218A.

33. Wanless IR. The mechanism of irreversibility of late stage cirrhosis. J Hepatology. 2018;68(suppl 1):S692.

34. Hano H, Takasaki S, Endo Y, Harada T, Komine K, Koike Y. Histological reassessment of the role of bridging fibrosis in the angioarchitectural features associated with lobular distortion of the liver in chronic viral hepatitis. Hepatol Res. 2016;46(3):E70-8.

35. Mall FP. A study of the structural unit of the liver. Am J Anat. 1906;5:18-308.

36. Wanless IR. Arterialization of the liver causes baro-injury in cirrhosis and focal nodular hyperplasia (FNH). Hepatology. 1999;30: 571A.

37. Wanless IR, Bernier V. Fibrous thickening of the splenic capsule. A response to chronic splenic congestion. Arch Pathol Lab Med. 1983;107(11):595-9.

38. Wanless IR, Liu JJ, Butany J. Role of thrombosis in the pathogenesis of congestive hepatic fibrosis (cardiac cirrhosis). Hepatology. 1995;21(5): 1232-7.

39. Mueller S. Does pressure cause liver cirrhosis? The sinusoidal pressure hypothesis. . World J Gastroenterol 2016;22:10482-501.

40. Frickhofen N, Wiesneth M, Jainta C, Hertenstein B, Heymer B, Bianchi $\mathrm{L}$, et al. Hepatitis $\mathrm{C}$ virus infection is a risk factor for liver failure from veno-occlusive disease after bone marrow transplantation. Blood. 1994;83(7):1998-2004.

41. Sabbagh C, Cosse C, Chauffert B, Nguyen-Khac E, Joly JP, Yzet T, et al. Management of colon cancer in patients with cirrhosis: a review. Surg Oncol. 2015;24(3):187-93. 
42. Marrone G, Maeso-Diaz R, Garcia-Cardena G, Abraldes JG, Garcia-Pagan JC, Bosch J, et al. KLF2 exerts antifibrotic and vasoprotective effects in cirrhotic rat livers: behind the molecular mechanisms of statins. Gut. 2015;64(9):1434-43.

43. Abraldes JG, Villanueva C, Aracil C, Turnes J, Hernandez-Guerra $\mathrm{M}$, Genesca J, et al. Addition of simvastatin to standard therapy for the prevention of variceal rebleeding does not reduce rebleeding but increases survival in patients with cirrhosis. Gastroenterology. 2016;150(5):1160-70 e3. Clinical trial indicating that simvastatin may influence the natural history of cirrhosis.

Publisher's Note Springer Nature remains neutral with regard to jurisdictional claims in published maps and institutional affiliations. 